\title{
Estimation of the shear strength of a coarse soil with high fines content by parallel gradation method
}

\author{
$N^{\prime}$ guessan Mö̈se Kouakou ${ }^{1,2^{*}}$, Olivier Cuisinier ${ }^{1}$, Farimah Masrouri ${ }^{1}$, Emmanuel Lavallée $^{2}$, and Tangi Le Borgne ${ }^{2}$ \\ ${ }^{1}$ LEMTA (UMR7563, Université de Lorraine - CNRS), 54500 Vandœuvre-lès-Nancy, France \\ ${ }^{2}$ Bouygues Travaux Publics, 78000 Guyancourt, France
}

\begin{abstract}
The determination of the mechanical properties of soils containing particles larger than the allowable size of standard laboratory equipments is complex. It is indeed necessary to remove the coarsest fraction to carry out the tests. This scalping poses a problem of reliability of the results at the scale of the structure. Parallel gradation is the method commonly used for estimating the shear strength of heterogeneous granular soils from tests on their finer fraction. However, the effect of high fines content on the estimation of shear strength by this method is not well understood. The results of this study show that the parallel gradation method can predict the friction angle of the initial soil with high fines content when the modelled soil has a similar skeleton as the initial soil. However, the cohesion of the initial soil is overestimated.
\end{abstract}

\section{Introduction}

Heterogeneous granular soils with matrix are composed of variable grains size (from a few microns to several tens of centimetres). Soil types in this category can be of natural deposit (alluvium, scree, moraine, etc.) or made by humans (rockfill). These soils are often used in the construction of dikes and embankments. Their applications in geotechnical projects require the determination of their mechanical characteristics in laboratory, which is a challenge due to the presence of coarse grains. Indeed, the maximum grain size of these soils is larger than the maximum size allowed by common laboratory shear devices. One solution is to use large devices. For example, a direct shear box of $1 \mathrm{~m}^{3}$ was used to determine the failure parameters of rock aggregates [1] and a triaxial cell of $1 \mathrm{~m}$ in diameter and $1.5 \mathrm{~m}$ in height was used for studying rockfills [2]. However, these devices, which allow to test soils with a grain size up to $160 \mathrm{~mm}$, are few, complex to implement and unsuitable for materials with larger grain size.

Therefore, some authors have developed methods to estimate with good accuracy the mechanical parameters of these soils from tests on their fraction compatible with standard laboratory shear equipments. Three modelling techniques have been proposed in the literature: substitution, scalping, and parallel gradation.

Among these approaches, parallel gradation is the most commonly used because it preserves the grain size distribution curve of the initial soil. It consists in scalping the oversize grains and prepares a material with a grain size distribution parallel to the initial soil. Using this method, the modelled material has a greater friction angle than the original material [3]. However, these authors showed that the friction angles of the two materials are identical for the same grain breakage ratio. Based on these results, an analytical method was proposed to take into account the effect of grain breakage during shearing and thus have a good estimate of the failure criterion of the initial material [4]. Furthermore, some authors concluded that the method leads to an underestimation of the initial soil shear strength when the breakage ratio is low and an overestimation when the breakage ratio is high [5]. In addition, most of the studies were carried out on materials with low fines content. Therefore, some questions remain about the effect of high fines content in the modelled material. For soils with high fines content, some authors proposed to attribute the fines content of the initial soil to the modelled soil. But a study shows that this underestimates the shear strength of the initial soil [6]. Therefore, even if promising results have been obtained by the parallel gradation method on coarse granular soils with matrix, the effect of higher fines content in the modelled soil remains unknown.

This study focuses on estimating the shear strength of heterogeneous granular soils with a high percentage of fines $(>10 \%)$ by using the parallel gradation method. The main question will be whether the parallel gradation method can give a good estimation of shear strength despite a high fines content in the initial material. Thus, the failure criteria of an initial soil with a high percentage of fines and two modelled soils obtained by parallel gradation method will be determined and compared.

\footnotetext{
* Corresponding author: n-guessan-moise.kouakou@ $@$ univ-lorraine.fr
} 


\section{Material and methods}

\subsection{Soils characteristics}

A natural soil with particles size up to $120 \mathrm{~mm}$ was chosen as the support soil for the study. It's containing $4.3 \%$ of fines (particles smaller than $0.080 \mathrm{~mm}$ ). Since the dimensions of the available equipments (see section 2.2) require a maximum grain size of $30 \mathrm{~mm}$, particles larger than $30 \mathrm{~mm}$ are removed. The $0-30 \mathrm{~mm}$ soil with grading curve parallel to the initial soil was chosen as the prototype soil for shear tests. Its maximum dry bulk density determined from a Proctor test is $21.1 \mathrm{kN} / \mathrm{m}^{3}$ for a water content of $8.3 \%$. From the prototype soil, two modelled soils with parallel grading curve were made (015 and $0-5 \mathrm{~mm}$ ). The grading curves of each material are shown in Figure 1. The fines content of 0-30, 0-15 and 0$5 \mathrm{~mm}$ specimens are $15 ; 19.8$ and $22.5 \%$ respectively.

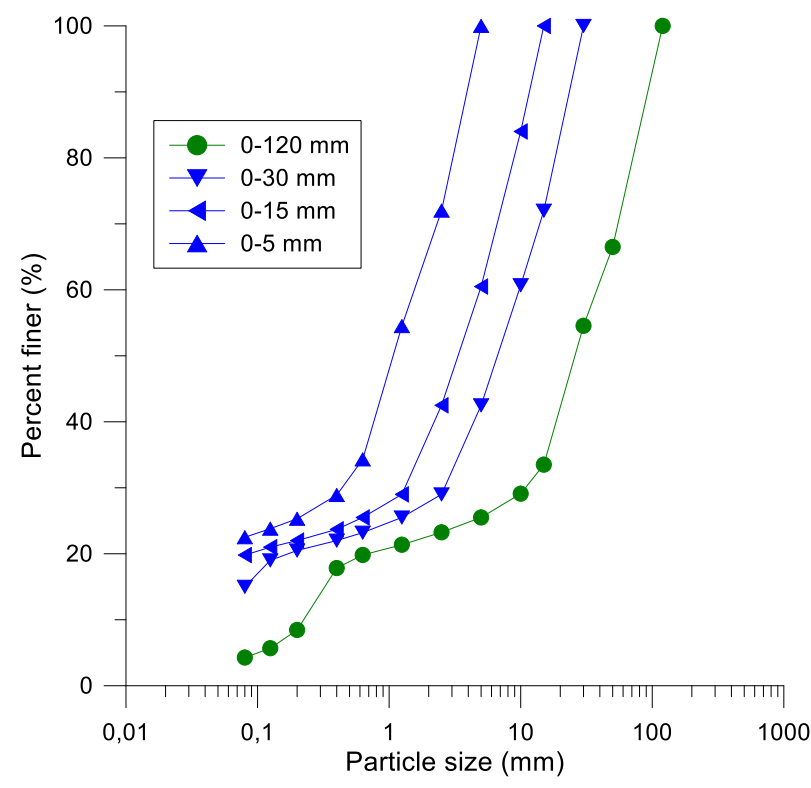

Fig. 1 : Grain size distributions of studied materials

\subsection{Direct shear apparatuses}

Three direct shear boxes were used: a small box of $60 \mathrm{x}$ $60 \times 45 \mathrm{~mm}^{3}$, a medium box of $150 \times 150 \times 180 \mathrm{~mm}^{3}$ and a large box of $300 \times 300 \times 180 \mathrm{~mm}^{3}$. The small box was used for $0-5 \mathrm{~mm}$ specimens, the medium box for $0-15 \mathrm{~mm}$ specimens and the large box for $0-30 \mathrm{~mm}$ specimens.

The small box is a classic direct shear box for soil mechanics.

The large direct shear box is composed of two half boxes: the lower half box is fixed while the upper half box is pushed by a motor at constant velocity. The crosssection of the large box can be reduced to $150 \times 150 \mathrm{~mm}^{2}$ using adapters to get the medium box.

A system of frame and hydraulic piston with a maximum capacity of $100 \mathrm{kN}$ applies a constant normal load during the test. Displacement sensors are installed to record the horizontal and vertical movements of the specimen. These sensors have a range of $50 \mathrm{~mm}$ with an accuracy of $0.2 \%$. The shear force is measured by a load sensor with a capacity of $100 \mathrm{kN}$. The normal load is measured by a load sensor placed between the frame and the upper plate. An inclination sensor installed on the upper plate records its inclination movements during the test. All the sensors are connected to a central acquisition unit which transmits the data to a software for recording and visualization.

\subsection{Experimental strategy}

The purpose of the tests is to know if the parallel gradation method can be used to predict the shear strength of coarse soil with high fines content. To do so, the modelled materials $0-5,0-15$ and $0-30 \mathrm{~mm}$ with grain size distribution parallel to the initial material were tested at the same dry density of $2 \mathrm{Mg} / \mathrm{m}^{3}$. The same density was chosen for all specimens for a better comparison of their shear strengths [4]. The failure criteria of these specimens were determined from consolidated drained tests and compared.

\subsection{Samples preparation}

In order to reconstitute the desired grain size for the specimens, the particles size up to $30 \mathrm{~mm}$ of the initial sample was separated into different fractions by sieving: $<0.08 ; 0.08-0.4 ; 0.4-0.63 ; 0.63-1.25 ; 1.25-2.5 ; 2.5-5 ; 5$ $10 ; 10-15$ and $15-30 \mathrm{~mm}$. The different fractions are mixed according to the proportions given by the grading curve for the preparation of the specimens. All the specimens are prepared at the optimal water content $(8.3 \%)$ and kept in airtight bags for at least 24 hours to ensure good homogeneity of the water in the samples. The specimens are then statically compacted until the desired density is reached: in three layers inside the medium or large shear box and in one layer inside the small box. The specimens were saturated by filling the shear apparatus tank with demineralised water and allowing it to flow through the sample for at least three hours. After saturation, the specimens are consolidated under the desired normal stress and then sheared at a constant rate of $0.05 \mathrm{~mm} / \mathrm{min}$

\section{Results}

\subsection{Parallel gradation stress-strain curves}

The stress-strain curves and the volumetric strain responses of the three specimens tested under different normal stresses are presented in Figures 2 to 4 . The stressstrain curves show a distinct peak and residual values for $0-5 \mathrm{~mm}$ specimen. In the case of $0-15$ and $0-30 \mathrm{~mm}$ specimens, there is no apparent peak while all specimens are tested at the same dry density. The absence of a peak for $0-15$ and $0-30 \mathrm{~mm}$ specimens could be explained by the large dimensions of the shear box used. Indeed, some authors have found that as the shear box dimensions increase, the difference between peak and residual strength decreases until it disappears completely [7, 8]. Also, the maximum shear values obtained for the $0-15$ 
$\mathrm{mm}$ specimen are substantially equal to the peak values for the $0-5 \mathrm{~mm}$ specimen, except under the stress of 100 $\mathrm{kPa}$. As for the 0-30 mm specimen, its maximum shear strengths are between the peak and residual values of the 0-5 mm specimen.
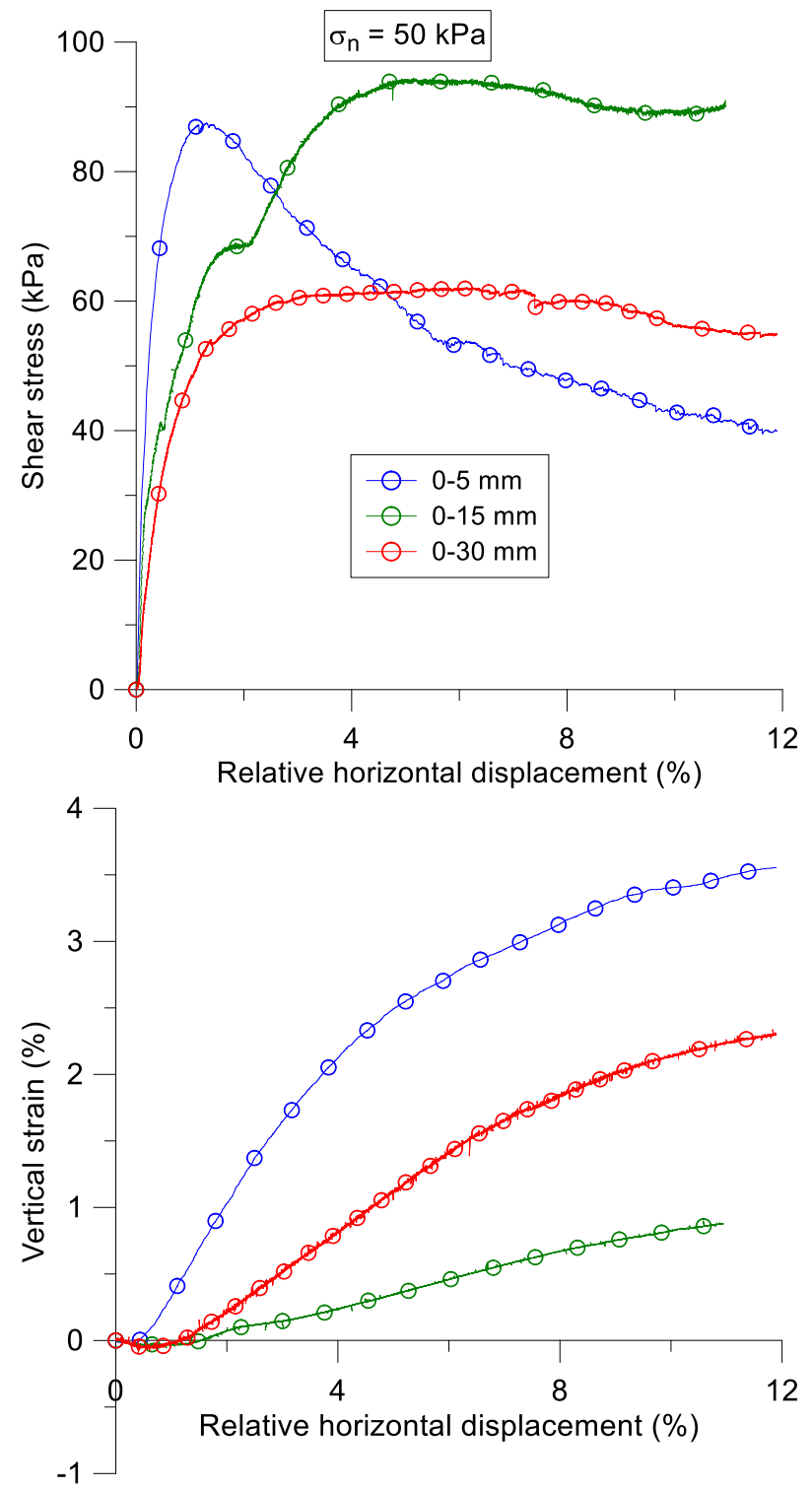

Fig. 2 : Stress-strain curves of studied samples under a normal stress of $50 \mathrm{kPa}$

The volumetric curves start with a contractance phase followed by a dilation phase characteristic of the dense state of the specimens in all boxes. The contractance of the $0-5 \mathrm{~mm}$ material is very low regardless the normal stress with a maximum value of $0.05 \%$ while it occurs over a longer deformation interval with a higher value for 0-15 and 0-30 $\mathrm{mm}$ specimens. However, the dilation has a higher value for $0-5 \mathrm{~mm}$ material compared to $0-30 \mathrm{~mm}$ material. The specimen $0-15 \mathrm{~mm}$ has lowest dilation values whereas they were expected between those of 0-5 and 0-30 $\mathrm{mm}$ specimens. This could be explained by the higher height/width ratio of the box for this specimen. Indeed, 0-5 and 0-30 $\mathrm{mm}$ specimens have a height/width ratio of about 0.6 while $0-15 \mathrm{~mm}$ specimen has a ratio of 1.1 .
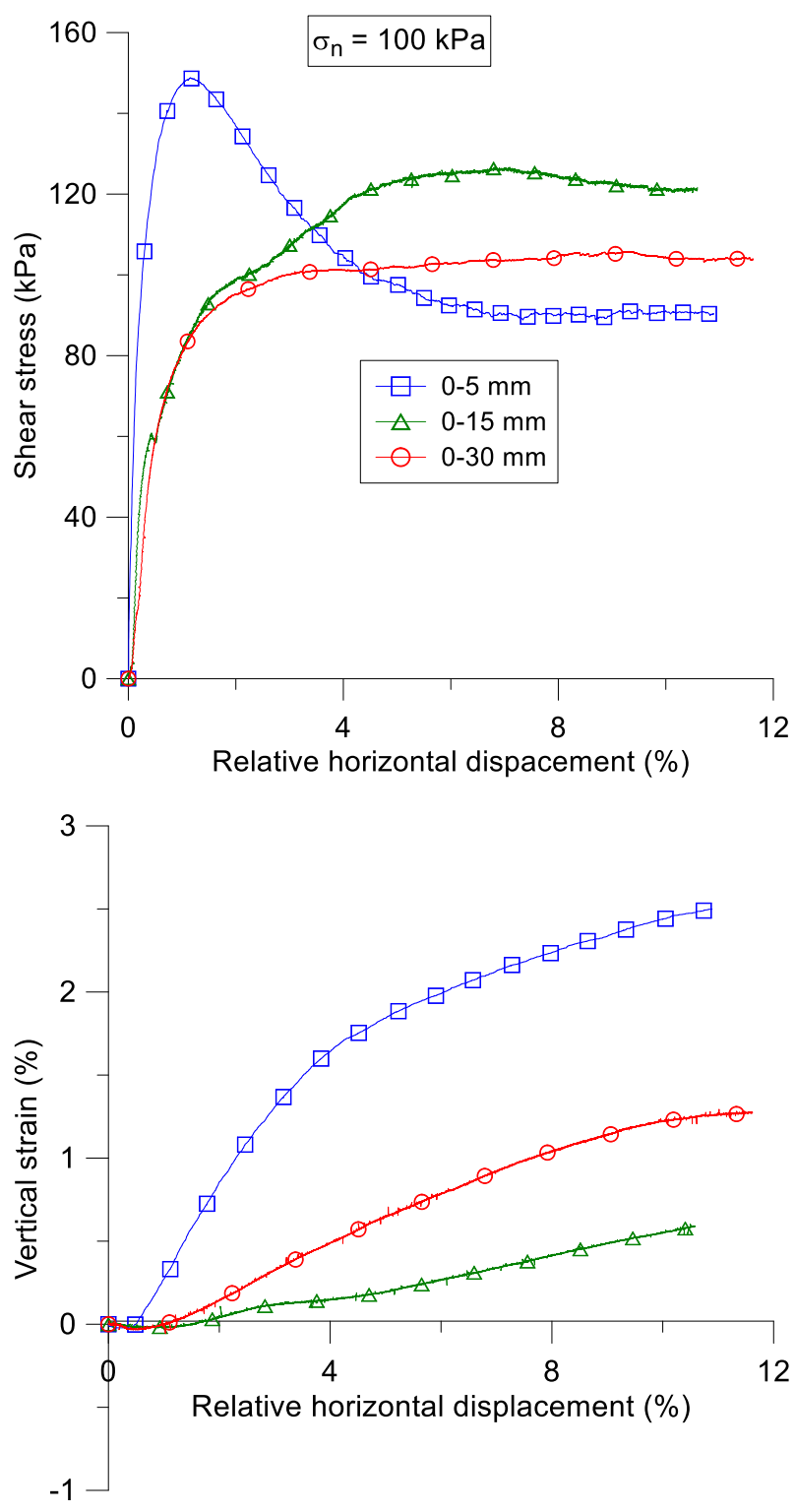

Fig. 3 : Stress-strain curves of studied samples under a normal stress of $100 \mathrm{kPa}$

\subsection{Failure criteria of studied materials}

The failure criteria for parallel grain size specimens according to the Mohr-Coulomb model are shown in Figure 5. The three specimens have a same maximum friction angle. The approach therefore gives a good prediction of the friction angle of coarse soils with high fines content in the range of applied stresses. The high fines content in the studied soil had no effect on the estimation of the friction angle by the method.

However, the cohesion increases when increasing fines content. Indeed, the finer particles create bonds between the granular ones. Therefore, there is an increase in soil cohesion when increasing fines content.

A result in literature shows that from a certain fines content in the modelled material, the parallel gradation method can no longer predict the angle of friction of the 
initial material [9]. Indeed, the authors tested two materials of parallel grain size: $0-25.4 \mathrm{~mm}$ specimen with $17 \%$ fines and $0-4.75 \mathrm{~mm}$ specimen with $33 \%$ fines. They found a higher cohesion and a lower friction angle for the 0-4.75 mm specimen. This difference is probably related to the difference in the skeleton of the two specimens. The $0-25.4 \mathrm{~mm}$ specimen has a skeleton imposed by the granular elements of the soil while the $0-4.75 \mathrm{~mm}$ specimen skeleton is partially controlled by fines. The parallel gradation method would therefore give a good estimation of the friction angle for a skeleton of the modelled soil identical to that of the initial soil, i.e. a fines content less than 20 to $35 \%[10,11]$.
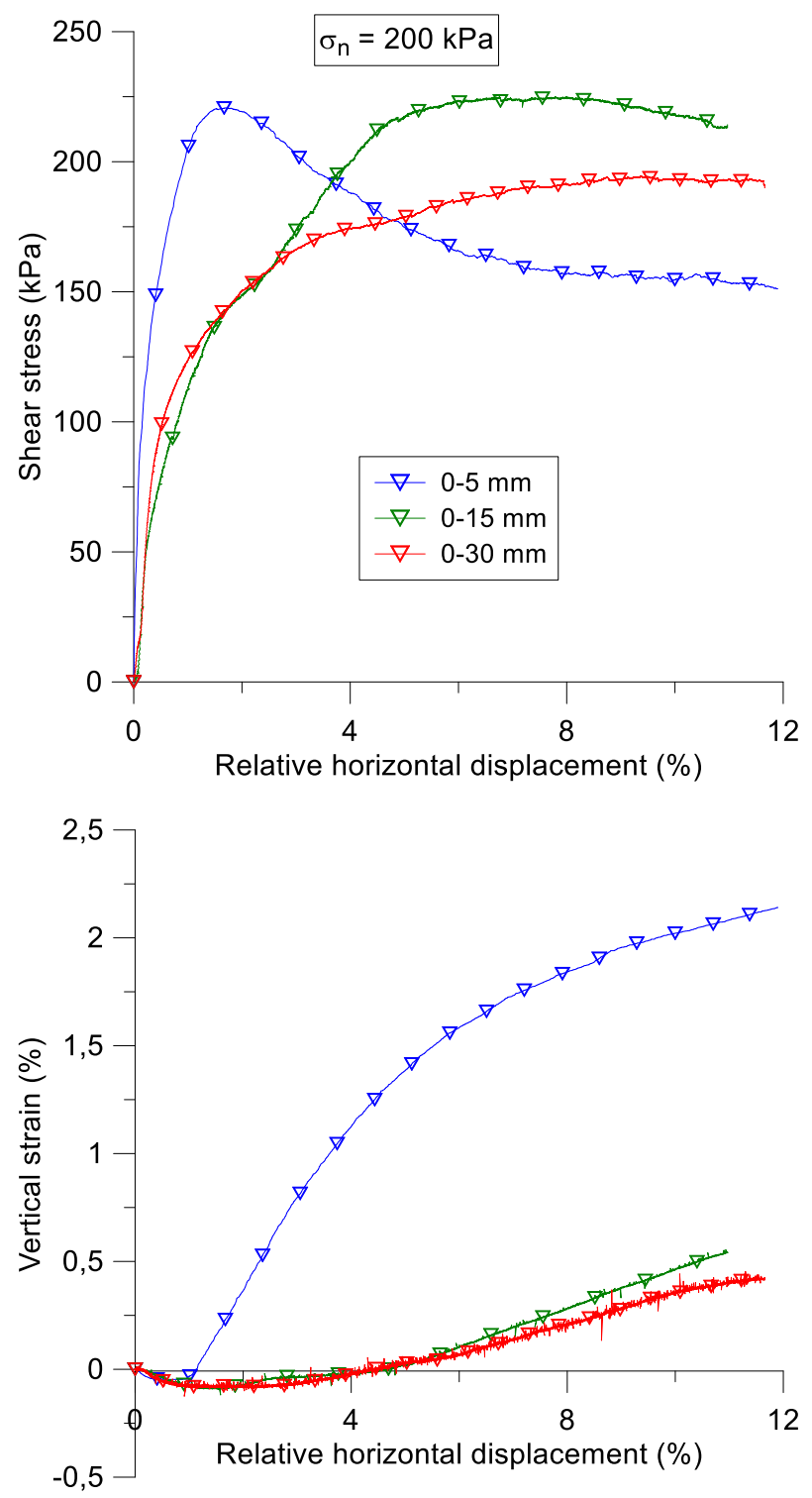

Fig. 4 : Stress-strain curves of studied samples under a normal stress of $200 \mathrm{kPa}$

Considering the obtained results and the fact that the tested materials have a grain size parallel to the $0-120 \mathrm{~mm}$ sample, an estimation of the failure parameters of the 0 $120 \mathrm{~mm}$ soil can be proposed. Thus, a friction angle of $41^{\circ}$ and zero cohesion can be attributed to the $0-120 \mathrm{~mm}$ soil because it contains low fines content $(4.3 \%)$.

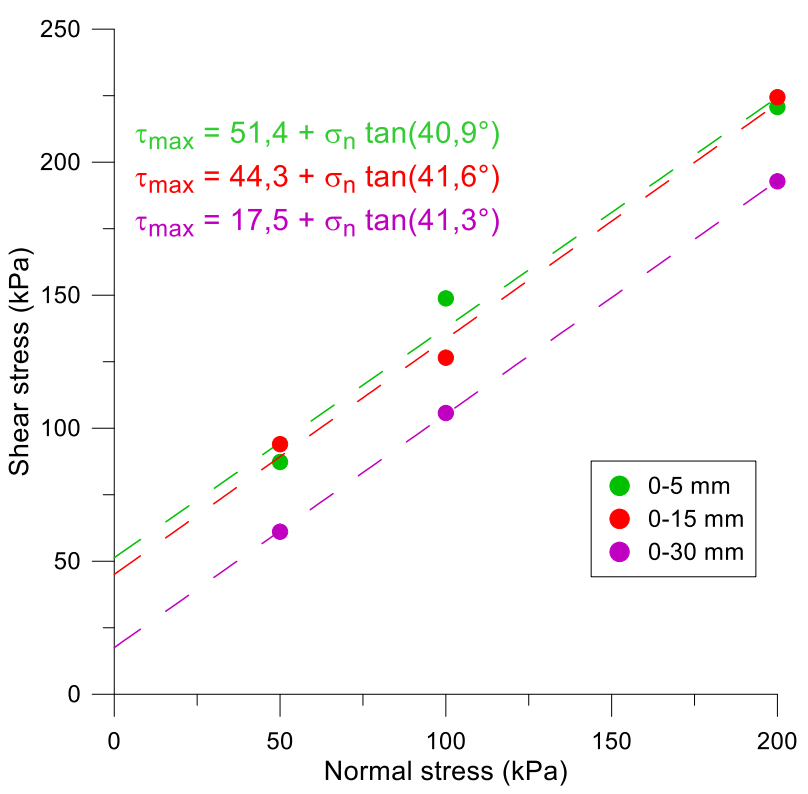

Fig. 5 : Shear strength envelopes for 0-5, 0-15 and 0-30 mm

\section{Conclusions}

The aim of this study was to determine if the shear strength of a granular soil with fines content greater than $10 \%$ can be correctly estimate by parallel gradation method. The main conclusion is:

The parallel gradation method gives a good estimation of the maximum friction angle of heterogeneous granular soils with high fines content when the modelled soil has a similar skeleton as the initial soil. However, the significant increase of fines content in the modelled soil leads to an overestimation of the cohesion.

Additional tests are needed to fully understand the evolution of soil skeleton when increasing fines content in the modelled soil.

\section{References}

[1] J. Estaire, C. Olalla, WIT Transact. on The Built Env., 78, (2005).

[2] C. Ovalle, E. Frossard, C. Dano, W. Hu, S. Maiolino, P.-Y. Hicher, Acta Mech., 225, 8, (2014).

[3] N. D. Marachi, C. K. Chan, H. B. Seed, J. of the Soil Mech. and Found. Div., 98, SM1, (1972).

[4] E. Frossard, W. Hu, C. Dano, P.-Y. Hicher, Géotechnique, 62, 5, (2012).

[5] A. Varadarajan, K. G. Sharma, K. Venkatachalam, A. K. Gupta, J. of Geotech. and Geoenv. Eng., 129, 3, (2003).

[6] R. Verdugo, K. De La Hoz, in Soil stress-strain behavior: Measurement, modeling and analysis, Roma, (2006).

[7] P. Gotteland, J. M. Tacnet, A. Aboura, Rev. Fr. de Génie Civ., 4, (2000).

[8] C. A. Bareither, C. H. Benson, T. B. Edil, Can. Geotech. J., 45, (2008).

[9] A. Bagherzadeh-Khalkhali, A. A. Mirghasemi, Particuology, 7, (2009). 
[10] R. J. Fragaszy, J. Su, F. H. Siddiqi, C. L. Ho, J. of Geotech. Eng., 6, 118, (1992).

[11] L. E. Vallejo, Can. Geotech. J., 38, 5, (2001). 